\title{
Deconvolution of ultrasound images
}

\author{
Jensen, Jørgen Arendt
}

Published in:

Ultrasonic Imaging

Publication date:

1992

Document Version

Publisher's PDF, also known as Version of record

Link back to DTU Orbit

Citation (APA):

Jensen, J. A. (1992). Deconvolution of ultrasound images. Ultrasonic Imaging, 14(1), 1-15.

\section{General rights}

Copyright and moral rights for the publications made accessible in the public portal are retained by the authors and/or other copyright owners and it is a condition of accessing publications that users recognise and abide by the legal requirements associated with these rights.

- Users may download and print one copy of any publication from the public portal for the purpose of private study or research.

- You may not further distribute the material or use it for any profit-making activity or commercial gain

- You may freely distribute the URL identifying the publication in the public portal

If you believe that this document breaches copyright please contact us providing details, and we will remove access to the work immediately and investigate your claim 


\title{
DECONVOLUTION OF ULTRASOUND IMAGES
}

\author{
Jørgen Arendt Jensen \\ Electronics Institute, bldg. 349 \\ Technical University of Denmark \\ DK-2800 Lyngby \\ Denmark
}

Based on physical models, it is indicated that the received pressure field in ultrasound B-mode images can be described by a convolution between a tissue reflection signal and the emitted pressure field. This result is used in a description of current image formation and in formulating a new processing scheme. The suggested estimator can take into account the dispersive attenuation, the temporal and spatial variation of the pulse, and the change in reflection strength and signal-to-noise ratio. Details of the algorithm and the estimation of parameters to be used are given. The performance is indicated by two examples. One is for a synthetic signal and the other is for data measured from a tissue mimicking phantom. The last example shows a finer speckle pattern, giving an increased resolution. 1992 Academic Press, Inc.

Key words: Deconvolution; estimation; image improvement; signal processing.

\section{INTRODUCTION}

Real-time B-mode ultrasound scanners show images of interior soft-tissue structures by employing the pulse-echo method. A short ultrasound pulse is emitted into the body and is reflected at boundaries between tissues with different characteristic impedance. A cross-sectional image is made by controlling the direction of the emitted ultrasound beam either mechanically (a rotating transducer element) or electronically (a phased array transducer).

The images are shown in real-time in order to perceive the relation between the position of the transducer and the location of the tissue structures, and to follow, e.g., heart valve movements. Often, 20 images are displayed per second, imposing a heavy demand on the speed of the signal processing involved. Consequently, the processing must be fast, enforcing a simple analog processing of the high frequency signal from the transducer. The signal processing essentially consists of a detection of the amplitude of the signal by rectification and low-pass filtering. This simple processing does not fully utilize the information present in the measured signal; most notably, the phase is ignored.

Diagnosis from ultrasound images is based on a number of features, including the displayed information about relative position and size of organs, and demarcations between tissues types. The accuracy of the diagnosis depends on the quality of the equipment, in terms of the capability to resolve tissue structures and the reproducibility from measurement to measurement.

Interpretation of the acquired images is often difficult and limits the application of ultrasound in the diagnostic work. A prime cause for this is the poor image quality. The images are blurred due to the finite signal bandwidth of the transducers, which limits the axial resolution.

Signals measured from current transducers contain information that is not fully utilized by currently used signal processing. This could be remedied by the use of more advanced digital 
signal processing. The aim of this research is to devise new digital methods to enhance the diagnostic value of ultrasound images.

It is shown in the next section that the received signal is generated by a convolution between the emitted pressure field and the tissue reflection signal. This knowledge is used in section to analyze the current image formation and to suggest a new algorithm for the removal of the pulse from the image. Details of the algorithm are given in section, and two examples of its use are shown.

\section{PROPAGATION OF ULTRASOUND IN TISSUE}

A three dimensional pulsed pressure field is emitted into the tissue during medical ultrasonic investigations. The field interacts with the tissue and part of it is reflected and scattered and subsequently received by the transducer.

The largest part of the received field emanates from scattering by small density and propaga tion velocity perturbations. This is in contrast to seismic oil exploration in which the dominant source of energy for the received field is from reflections due to the different characteristic acoustic impedances of the layers.

In medical ultrasound, it is rare to find pure reflections from surfaces perpendicular to the direction of propagation. Often, smooth boundaries do not exist, or are oblique to the propagation direction, so the reflected field is not directed towards the transducer, and thus no response is received. Rather, small surface roughness or local changes in density and propagation velocity give rise to an emitted spherical wave that is received by the transducer. Tissue structures are therefore indicated instead by the speckle pattern, so boundaries are seen as a collection of small speckle spots. Thus, it is appropriate to analyze the scattering mechanisms and relate the backscattered field to the emitted field and to the transducer reception response and geometry.

To analyze the situation, the following wave equation was derived when absorption was neglected and linear propagation and weak scattering assumed [1-3]:

$$
\nabla^{2} p_{1}-\frac{1}{c_{0}^{2}} \frac{\partial^{2} p_{1}}{\partial t^{2}}=-\frac{2 \Delta c}{c_{0}^{3}} \frac{\partial^{2} p_{1}}{\partial t^{2}}+\frac{1}{\rho_{0}} \nabla(\Delta \rho) \cdot \nabla p_{1}
$$

$p_{1}$ is the overpressure, $\nabla$ the gradient and $\nabla^{2}$ the Laplace operator. $c_{0}$ is the propagation velocity, $\rho_{0}$ the mean density and $\Delta c, \Delta \rho$ the corresponding perturbations in the scattering region.

Neglecting multiple scattering and introducing the Tupholme-Stepanishen model for the pressure field from the transducer, results in the following equation for the received pressure field [1], [3]:

$$
p_{r}\left(\vec{r}_{2}, t\right)=v_{p e}(t) \underset{t}{\star} f_{m}\left(\vec{r}_{1}\right) \underset{r}{\star} h_{p e}\left(\vec{r}_{1}, \vec{r}_{2}, t\right)
$$

where ${ }_{t}^{\star}$ denotes a temporal and ${ }_{r}^{\star}$ a spatial convolution. $v_{p e}$ is the pulse-echo wavelet that accounts for the transducer excitation and the electromechanical impulse response during emission and reception of the pulse. $f_{m}$ accounts for inhomogeneities in the tissue due to density and propagation velocity perturbations, which gives rise to the scattered signal. $h_{p e}$ is the modified pulse-echo spatial impulse response that relates the transducer geometry to the spatial extent of the scattered field. Explicitly written out, these terms are:

$$
\begin{gathered}
v_{p e}(t)=\frac{\rho_{0}}{2} E_{m}(t) \star \frac{\partial v(t)}{\partial t} \\
f_{m}\left(\vec{r}_{1}\right)=\frac{\Delta \rho\left(\vec{r}_{1}\right)}{\rho_{0}}-\frac{2 \Delta c\left(\vec{r}_{1}\right)}{c_{0}} \\
h_{p e}\left(\vec{r}_{1}, \vec{r}_{2}, t\right)=\frac{1}{c_{0}^{2}} \frac{\partial h\left(\vec{r}_{1}, \vec{r}_{2}, t\right)}{\partial t} \star \frac{\partial h\left(\vec{r}_{1}, \vec{r}_{2}, t\right)}{\partial t}
\end{gathered}
$$


$\vec{r}_{1}$ denotes the position of the scatterer and $\vec{r}_{2}$ the position of the transducer. $v(t)$ is the transducer excitation and electromechanical impulse response and $E_{m}(t)$ the impulse response when receiving. $h$ is the spatial impulse response for the transducer geometry as calculated by the Tupholme-Stepanishen method [4-6]. Equation (2) was derived under the assumption of weak scattering due to density and propagation velocity perturbations in the tissue, and absorption and multiple scattering were neglected.

Expression (2) consists of three distinct terms. The signal of interest, and the one that should be displayed in medical ultrasound, is $f_{m}\left(\vec{r}_{1}\right)$. We, however, measure a time and spatially smoothed version of this, which obscures the finer details in the image. The smoothing consists of a convolution in time with a fixed wavelet $v_{p e}(t)$, and a spatial convolution with a spatially varying $h_{p e}\left(\vec{r}_{1}, \vec{r}_{2}, t\right)$.

Equation (2) does not included the dispersive attenuation in the wave equation describing the pulse. This would have the effect of continuously changing the pulse as it propagates through the tissue. Neglecting dispersive attenuation is, however, not a serious drawback of the theory, as this change of the pulse can be lumped into the already spatially varying $h_{p e}$.

A second approach is to assume a homogeneous absorption, and propagation in the far field. Then the absorption, $A\left(t,\left|\vec{r}_{1}-\vec{r}_{2}\right|\right)$, can be lumped into $v_{p e}$ to yield an attenuated pulse:

$$
v_{\text {pea }}\left(t,\left|\vec{r}_{1}-\vec{r}_{2}\right|\right)=v_{p e}(t) \underset{t}{\star} A\left(t,\left|\vec{r}_{1}-\vec{r}_{2}\right|\right)
$$

that is a function of time and distance.

\section{ULTRASOUND B-SCAN IMAGING: $\Lambda$ N ESTIM $\Lambda$ TION-BASED APPROACH}

In the preceding section, we saw that the received signal can be written in a convolution form. This result will here be used to analyze the image formation in traditional B-mode systems and to suggest a new improved estimation of the amplitude reflection signal. It will consist of adaptive axial deconvolution and adaptive two-dimensional deconvolution.

\subsection{Current B-scan systems}

The following expression was given for the received signal:

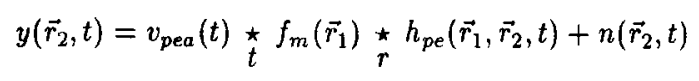

A noise term $n\left(\vec{r}_{2}, t\right)$ has been added to explain the inevitable noise in the measured signal. The term accounts for both electrical noise from amplifiers and for physical effects not explained by the convolution model.

In a B-mode system, a number of lines is collected and combined to an image. The signal displayed on the screen is:

$$
s_{d}\left(\overrightarrow{r_{2}}, t\right)=\left|y\left(\vec{r}_{2}, t\right)\right| \underset{t}{\star} h_{l p}(t)
$$

$y\left(\vec{r}_{2}, t\right)$ is rectified and low-pass filtered to avoid aliasing in the subsequent sampling process. $h_{l p}$ denotes the low-pass filter used. $s_{d}\left(\vec{r}_{2}, t\right)$ is displayed on the screen, where the intensity is proportional to the amplitude of $s_{d}$.

The displayed signal is a rectified, smoothed, and noisy version of $f_{m}\left(\vec{r}_{1}\right) \cdot s_{d}\left(\vec{r}_{2}, t\right)$ approaches a true version of the rectified $f_{m}\left(\vec{r}_{1}\right)$, if the spatial extent of the object is large compared to the wavelength of the ultrasound, and the noise amplitude is small compared to the amplitude of $f_{m}\left(\vec{r}_{1}\right)$. The current resolution limit is 1 to 2 oscillations in the axial direction, primarily determined by $v_{\text {pea }}(t)$. In the lateral direction, it is 5 to 10 ascillations, determined by the 


\section{J.A. JENSEN}

transducer center frequency and $h_{p e}\left(\vec{r}_{1}, \vec{r}_{2}, t\right)$. This corresponds to $0.4-1 \mathrm{~mm}$ axially, and 2 to $4 \mathrm{~mm}$ laterally for a transducer center frequency of $4 \mathrm{MHz}$.

As is apparent, this method for estimating $f_{m}\left(\vec{r}_{1}\right)$ precludes obtaining any information about the sign of $f_{m}$. The reason for using this estimator is of course its simplicity, which makes it easy to implement. Real-time processing of signals can be accomplished as it can be implemented by analog electronics.

\subsection{A new estimation-based approach}

The problem of calculating $f_{m}\left(\vec{r}_{1}\right)$ is often called the inverse scattering problem in the literature $[8,9]$. Some solutions to this problem rely on purely deterministic approaches, which can result in gross errors due to modeling errors or noise in the data used for the reconstruction. In this paper, the problem is approached from a statistical point of view. So, it is stated as estimating the lissue inhomogeneities $f_{m}$ from the measured data $y$.

It is necessary to employ digital signal processing to make a better estimation of $f_{m}$. Thus, Eq. (7) is reformulated in a discrete form:

$$
y\left(\vec{r}_{d_{2}}, k\right)=v_{p e a}(k) \underset{k}{\star} f_{m}\left(\vec{r}_{d_{1}}\right) \underset{r_{d}}{\star} h_{p e}\left(\vec{r}_{d_{1}}, \vec{r}_{d_{2}}, k\right)+n\left(\vec{r}_{d_{2}}, k\right)
$$

$k$ is the discrete time variable, and $\vec{r}_{d}$ is a discrete vector. [7]:

We now seek a procedure to obtain an estimate of $f_{m}\left(\vec{r}_{d_{1}}\right)$. This can formally be stated as

$$
\hat{f}_{m}\left(\vec{r}_{d_{1}}\right)=\mathcal{F}\left[y\left(\vec{r}_{d_{2}}, k\right), v_{p e a}(k), h_{p e}\left(\vec{r}_{d_{1}}, \vec{r}_{d_{2}}, k\right), n\left(\vec{r}_{d_{2}}, k\right)\right]
$$

The estimate $\hat{f}_{m}\left(\vec{r}_{d_{1}}\right)$ is a function of the measured signal $y\left(\vec{r}_{d_{2}}, k\right)$ and some knowledge, measured or estimated, about $v_{p e a}, h_{p e}$, and $n$.

The function $\mathcal{F}$ can be a linear or nonlinear mapping from $y, v_{p e a}, h_{p e}$, and $n$ to $\hat{f}_{m}$. It can be evaluated directly or through iterative techniques. One of the advantages of ultrasound B-mode systems is the real-time image formation. This precludes the iterative search scheme, as in a real-time system only a fixed amount of calculations can be carried out per sample. Rather, the estimate $\hat{f}_{m}\left(\vec{r}_{d_{1}}\right)$ is based on some estimate of the internal state of the system so that

$$
\begin{aligned}
\hat{S}\left(\vec{r}_{d_{2}}, k\right) & =\mathcal{H}\left[y\left(\vec{r}_{d_{2}}, k\right), v_{p e a}(k), h_{p e}\left(\vec{r}_{d_{1}}, \vec{r}_{d_{2}}, k\right), n\left(\vec{r}_{d_{2}}, k\right)\right] \\
\hat{f}_{m}\left(\vec{r}_{d_{1}}\right) & =\mathcal{G}\left[\hat{S}\left(\vec{r}_{d_{2}}, k\right)\right]
\end{aligned}
$$

where $\mathcal{H}$ linearly relates $y\left(\vec{r}_{d_{2}}, k\right), v_{\text {pea }}(k), h_{p e}\left(\vec{r}_{d_{1}}, \vec{r}_{d_{2}}, k\right), \quad$ and $n\left(\vec{r}_{d_{2}}, k\right)$ to $\hat{S}\left(\vec{r}_{d_{2}}, k\right)$, and $\mathcal{G}$ linearly relates $\hat{S}\left(\vec{r}_{d_{2}}, k\right)$ to $\hat{f}_{m}\left(\vec{r}_{d_{1}}\right) . S$ is a vector of fixed dimension that represents the state of the system. $\mathcal{H}$ and $\mathcal{G}$ can be evaluated with a fixed and a priori known number of calculations. This allows for a time-varying model, where each sample can be treated in a fixed, predetermined amount of time [7].

From the calculation of the received signal, it is known that the smoothing of $f_{m}$ is due to convolution with $v_{p e a}$ and $h_{p e} . v_{p e a}$ is a one-dimensional entity, that is common to all A-lines in the image; thus, the estimation can advantageously be split into a two-part procedure. The first part is:

$$
\begin{aligned}
\hat{S}_{1}\left(\vec{r}_{d_{2}}, k\right) & =\mathcal{H}_{1}\left[y\left(\vec{r}_{d_{2}}, k\right), v_{\text {pea }}(k), n\left(\vec{r}_{d_{2}}, k\right)\right] \\
\hat{f}_{m_{1}}\left(\vec{r}_{d_{1}}\right) & =\mathcal{G}_{1}\left[\hat{S}_{1}\left(\vec{r}_{d_{2}}, k\right)\right]
\end{aligned}
$$


In this estimation step, the effect of smoothing due to $v_{\text {pea }}$ is removed. So, in the first part of the estimation procedure $v_{p e a}$ is estimated for one A-line, and it is used for the whole image. This is a one-dimensional operation, which is common to all A-lines.

The next part of the algorithm removes the (mainly lateral) smoothing by $h_{p e}$. This can be written:

$$
\begin{aligned}
\hat{S}_{2}\left(\vec{r}_{d_{1}}, k\right) & =\mathcal{H}_{2}\left[\hat{f}_{m_{1}}\left(\vec{r}_{d_{1}}\right), h_{p e}\left(\vec{r}_{d_{1}}, \vec{r}_{d_{2}}, k\right), n\left(\vec{r}_{d_{2}}, k\right)\right] \\
\hat{f}_{m}\left(\vec{r}_{d_{1}}\right) & =\mathcal{G}_{2}\left[\hat{S}_{2}\left(\vec{r}_{d_{1}}, k\right)\right]
\end{aligned}
$$

In this part, both the modified pulse-echo spatial impulse response and the signal-to-noise ratio must be continuously estimated.

Both Eq. (12) and (13) can be viewed as deconvolution operations [11], where Eq. (12) is a one-dimensional deconvolution and Eq. (13) a two-dimensional deconvolution. The next section will describe how these deconvolution operations are performed.

\section{ESTIMATING THE AMPLITUDE REFLECTION SIGNAL}

Our task is to derive a two-dimensional deconvolution algorithm for the general two-dimensional convolution between an excitation, $R(j, i)$ and a data channel $V(j, i)$ :

$$
z(k, h)=\sum_{j=1}^{M} \sum_{i=1}^{N} R(j, i) V(k-j, h-i)+n(k, h)
$$

$R$ represents the scattering signal ( $f_{m}$ in the previous sections), and $V$ is the two-dimensional spatial and time-varying wavelet. It can include both $v_{p e a}$ and $h_{p e}$ in Eq. (9) and the effect of the dispersive attenuation of the intervening tissue. $n$ is a noise term which accounts for the effects that are not included in the convolution model. In the remaining part of the paper, we will only write $z(k)$, which indicates a vector of samples from the different A-lines at time $k$.

It might seem physically counterintuitive that $R$ is the excitation and $V$ the system, but this is convenient from an estimation point of view. It might ease the understanding if $V$ is viewed as a time-varying data channel conveying information about the tissue, and the purpose of the estimation is to remove the effect of this nonideal data channel. The estimation procedure is then a deconvolution operation, which uses an optimal inverse filter to remove the effect of $V$ and $n$.

The purpose is to obtain an estimate of the amplitude reflection signal based upon all the obtained information. Thus, $\hat{R}(k \mid N)$ is estimated when $k=1 . . N$. The estimate is based on all the measurements $\mathcal{Z}(N)=\{z(1), z(2) \ldots z(N)\}$, where $z(N)$ denotes the measurement of all A-lines in the image at time $n . R(k \mid N)$ is related to $\mathcal{Z}(N)$ by some function $\varphi_{k}$ :

$$
\hat{R}(k \mid N)=\varphi_{k}(\mathcal{Z}(N))
$$

where $\varphi_{k}$ is time-varying. $\varphi_{k}$ is found by minimizing some criteria. We here choose to use the minimum-variance criterion:

$$
J_{1}(R(k) \mid \mathcal{Z}(N))=E\left[(R(k)-\hat{R}(k \mid N))^{T}(R(k)-\hat{R}(k \mid N))\right]
$$

$E$ denotes expectation, and $(\cdot)^{T}$ is the transposed.

This criterion gives a linear estimator [11], which is a very important property. It means that we do not have to use an iterative search scheme, and consequently a fixed, predeterminable 
amount of calculations is performed for each sample. This makes it, at least in theory, possible to obtain a real-time image formation.

Several authors have devised algorithms to estimate the amplitude reflection signal for ultrasound signals. These algorithms have mainly been based on the Wiener filter [12-15]. Slightly different, but conceptually similar methods have been suggested by Hundt \& Trautenberg [16], Herment et al. [17], Vollmann [18], Demoment et al. [19]. These estimators use, however, a fixed $\varphi_{k}$ and cannot take into account the spatially-varying source wavelet and the dispersive attenuation. Kuc [20] used Mendel's $l$-step ahead deconvolution algorithm. This uses $l$ future values of the A-line, and can handle time-varying parameters. We will carry this one step further indicating how to use all available samples in the A-line, and showing how to obtain parameters for the pulse and the signal-to-noise ratio directly from the data measured.

The algorithm used is Mendel's fixed-interval deconvolution estimator [10,11]. It consists of two parts: first a Kalman filtering performed on the data time recursively and then a subsequent estimation step performed backwards time-recursively. The result is a minimum variance, fixedinterval estimate of the reflection signal.

The estimator is based on a state-space model:

$$
\begin{aligned}
x(k+1) & =\Phi(k+1, k) x(k)+\Gamma(k+1, k) w(k+1) \\
z(k) & =H^{T}(k) x(k)+n(k)
\end{aligned}
$$

The dimension of the vectors and matrices are:

$$
\begin{gathered}
x-n \times 1 \\
\Phi-n \times n \\
\Gamma-n \times p \\
w-p \times 1 \\
z-m \times 1 \\
H^{T}-m \times n \\
n-m \times 1
\end{gathered}
$$

$p$ is the number of input channels (A-lines), $n$ is the order of the system, and $m$ the number of output channels.

$z(k)$ is the measured signal, and $n(k)$ is the measurement noise, which is assumed white, zero mean and has the covariance $R_{n}(k) . x(k)$ is the state vector and $w(k)$ is a stochastic disturbance acting on the states. $w(k)$ is equivalent to $R(k)$ and is assumed to be white, zero mean, and Gaussian with the covariance $Q(k)$. $\Phi$ is the state transition matrix, $\Gamma$ the input distribution matrix and $H$ the observation matrix. Note that the system is time varying and describes a two-dimensional system. The state-space model corresponds to a convolution model for fixed matrices $\Phi, \Gamma$ and $H[11]$.

The Kalman filter is, stated in the predictor-corrector form [11]:

\section{Predictor equations}

$$
\begin{aligned}
\hat{x}(k+1 \mid k)= & \Phi(k+1, k) \hat{x}(k \mid k) \\
P(k+1 \mid k)= & \Phi(k+1, k) P(k \mid k) \Phi^{T}(k+1, k)+ \\
& \Gamma(k+1, k) Q(k+1) \Gamma^{T}(k+1, k)
\end{aligned}
$$




\section{Corrector equations}

$$
\begin{aligned}
K(k+1)= & P(k+1 \mid k) H(k+1) . \\
& {\left[H^{T}(k+1) P(k+1 \mid k) H(k+1)+R_{n}(k+1)\right]^{-1} } \\
\tilde{z}(k+1 \mid k)= & z(k+1)-H^{T}(k+1) \hat{x}(k+1 \mid k) \\
\hat{x}(k+1 \mid k+1)= & \hat{x}(k+1 \mid k)+K(k+1) \tilde{z}(k+1 \mid k) \\
P(k+1 \mid k+1)= & {\left[I-K(k+1) H^{T}(k+1)\right] P(k+1 \mid k) }
\end{aligned}
$$

$P(k+1 \mid k)$ is the a priori state covariance, and $P(k+1 \mid k+1)$ the a posteriori covariance. $K(k)$ is the Kalman gain.

The backwards time-recursive estimation step can be expressed as [11]:

$$
\begin{aligned}
\hat{w}(k+1 \mid N)= & Q(k+1) \Gamma^{T}(k+1, k) r(k+1 \mid N) \\
r(k \mid N)= & H(k)\left[H^{T}(k) P(k \mid k-1) H(k)+R_{n}(k)\right]^{-1} \tilde{z}(k \mid k-1) \\
& +\left(\Phi(k+1, k)\left[I-K(k) H^{T}(k)\right]\right)^{T} r(k+1 \mid N)
\end{aligned}
$$

$\hat{w}(k+1 \mid N)$ denotes the fixed-interval estimate of $w(k+1) \cdot r(k+1 \mid N)$ is the residual state vector [11]. The covariance for $\hat{w}(k+1 \mid N)$ is

$$
\Psi_{w}(k+1 \mid N)=Q(k+1)-Q(k+1) \Gamma^{T}(k+1, k) S(k+1 \mid N) \Gamma(k+1, k) Q(k+1)
$$

This algorithm can estimate the amplitude reflection signal from the noisy backscattered signal. It calculates a minimum-variance fixed-interval estimate of the reflection signal; thus, it uses all the available information in the acquired A-lines, and can be used for a two-dimensional, time-varying pulse.

\section{PULSE AND COVARIANCE ESTIMATION}

The basic in vivo pulse-echo wavelet must be known in order to perform a deconvolution as described previously. A prediction error algorithm was used in [21] to estimate a set of ARMA parameters for the pulse. Using it on data from a tissue mimicking phantom, and on a calf's liver, it was shown that the basic attenuated pulse can be estimated. 'The algorithm also yields an estimate of the reflection covariance $Q$. So, for a one dimensional deconvolution, the pulse estimator gives all the necessary parameters for performing the deconvolution. This makes the approach essentially self-calibrating as all parameters for the procedure estimated from the actual patient under investigation. A derivation of the algorithm and a number of examples of its use can be found in [1], [21].

The result from the wavelet estimation is a set of parameters for an ARMA model, which are used in a state-space model. Different mappings from the ARMA parameters to the state-space matrices exist, and we choose to use the controllable canonical form [11]. Here, the entries in the state-space matrices are equal to the ARMA parameters, and the mapping can always be used regardless of whether the model has real or complex poles and zeros.

For a single input single output ARMA model, we have:

$$
\left(1+a_{1} q^{-1}+a_{2} q^{-2} \cdots+a_{n} q^{-n}\right) y(k)=\left(1+c_{1} q^{-1}+\cdots+c_{n} q^{-(n-1)}\right) e(k)
$$




\section{J.A. JENSEN}

Then the state-space matrices and vectors are:

$$
\begin{aligned}
\Phi & =\left(\begin{array}{cccccc}
0 & 1 & 0 & 0 & \cdots & 0 \\
0 & 0 & 1 & 0 & \cdots & 0 \\
\vdots & & & \ddots & & \\
-a_{n} & a_{n-1} & \cdots & & -a_{2} & -a_{1}
\end{array}\right) \\
\Gamma & =\left(\begin{array}{c}
0 \\
\vdots \\
0 \\
1
\end{array}\right) \\
H^{T} & =\left(c_{n}, c_{n-1}, \cdots c_{2}, c_{1}, 1\right)
\end{aligned}
$$

\subsection{Initialization of the Kalman filter}

Before the Kalman filter can be used, initial values for the state vector, covariance matrix and Kalman gain, $x(0), P(0 \mid 0), K(0)$ must be supplied. A simple solution is to set $x(0)$ equal to the mean value of $x$ (normally zero), $P$ to a unit matrix multiplied by some large value reflecting our unreliable knowledge of $x$, and $K$ to zero.

If the state-space model is stable, which is the case when the absolute value of the eigenvalues of $\Phi$ are less than one, or correspondingly that the poles of the ARMA model are inside the unit circle, then $P$ and $K$ will become equal to their steady-state values [22]. This is of course under the assumption of a steady-state system, in which $\Phi, \Gamma, H, Q$ and $R_{n}$ are fixed. In this case, the error covariance matrix, $P(k+1 \mid k)$, will reach the steady state value, $\bar{P}$, determined by [11]

$$
\bar{P}=\Phi \bar{P}\left[I-H\left(H^{T} \bar{P} H+R_{n}\right)^{-1} H^{T} \bar{P}\right] \Phi^{T}+\Gamma Q \Gamma^{T}
$$

$\bar{P}$ is the solution to this matrix Riccati equation.

Experiments performed in this work have revealed that this steady state value for $P(k+1 \mid k)$ is reached to a good approximation after 20 to 30 time steps of the Kalman filter. Therefore, we here set $x(0)$ equal to zero, $K(0)$ to zero and $P(0 \mid 0)$ to the unit matrix. Then, 30 time steps of the Kalman filter are performed and the resulting $P(k \mid k)$ and $K(k)$ are then used as the initial values in the examples shown in this paper.

\section{DECONVOLUTION OF SYNTHETIC SIGNALS}

To show the performance of the combined algorithm, an example for a synthetic signal is given.

A synthetic reflection sequence of 500 samples was generated. It consists of both positive and negative spikes of varying amplitude. To compare the different results, a segment of 100 samples, between samples 201 and 300 , is used. Thus, zero on the $x$-axis corresponds to sample 201 on the following figures.

The input signal to the deconvolution algorithm was generated by convolving the pulse with the reflection signal, and then adding white, zero mean Gaussian noise. An example for a signal-to-noise ratio of 100 for the segment is shown in figure 1 .

The wavelet used in this example was estimated from a calf's liver, where the data were measured by a Brüel \& Kjær $85293 \mathrm{MHz}$ transducer. The parameters are:

$$
\begin{aligned}
A(q)= & 1.0000-4.4115 q^{-1}+8.8400 q^{-2}-10.1710 q^{-3} \\
& +7.1062 q^{-4}-2.8717 q^{-5}+0.5358 q^{-6}
\end{aligned}
$$




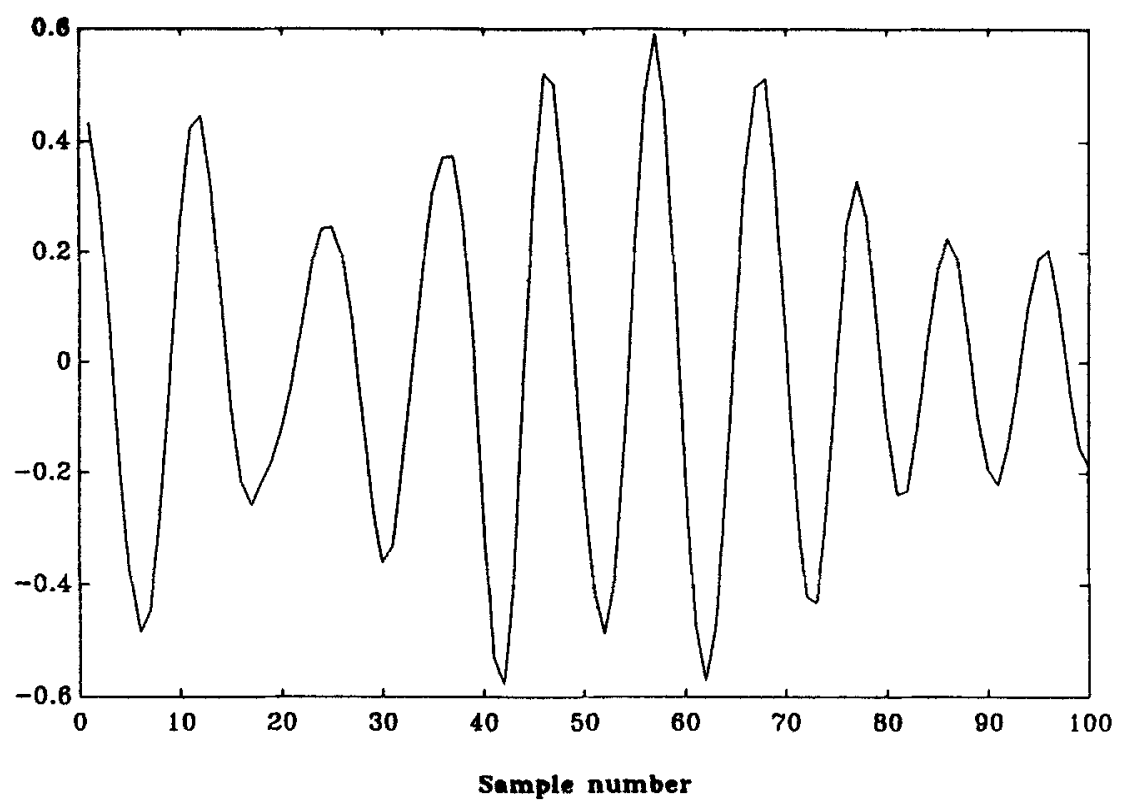

Fig. 1 Input signal to the deconvolution algorithm. $S N R=100$.

$$
\begin{aligned}
C(q)= & 1.000-2.7925 q^{-1}+3.2911 q^{-2}-2.6017 q^{-3} \\
& +1.6405 q^{-4}-0.5286 q^{-5}
\end{aligned}
$$

The signal-to-noise ratio is defined as:

$$
\mathrm{SNR}=\sqrt{\frac{E\left[y(k)^{2}\right]}{E\left[n(k)^{2}\right]}}
$$

where $y$ is the filtered signal and $n$ the noise. $E\left[{ }^{2}\right]$ is the covariance.

Using the parameter estimator and then the deconvolution algorithm on a synthetic signal with $\mathrm{SNR}=100$, we get the estimated wavelets shown in figure 2 and the estimated reflection signals shown in figure 3 . The dashed and solid lines are the true and estimated responses, respectively. The sampling frequency was $25 \mathrm{MHz}$, so one sample equals a distance in tissue of $0.03 \mathrm{~mm}$, assuming a propagation velocity of $1500 \mathrm{~m} / \mathrm{s}$.

The estimate of the wavelet is quite precise and all major reflections in the segments are identified with a good estimate of their strength and with the correct sign. Smaller reflections below 0.005 disappear in noise, as the estimator increases the noise in order to enhance resolution. Overall, we see that the resolution is approximately 1 to 2 samples, a considerable improvement, to the resolution of 10 to 20 samples.

\subsection{Deconvolution of data from a tissue phantom}

We will now show a result obtained by deconvolving signals measured from a tissue phantom. As we currently have no model for the pulse-echo spatial impulse response, only a $1 \mathrm{D}$ deconvolution will be performed with a fixed, estimated wavelet. As the data segment only spans a relatively small depth, a fixed value of $Q$ and $R_{n}$ will be used. 


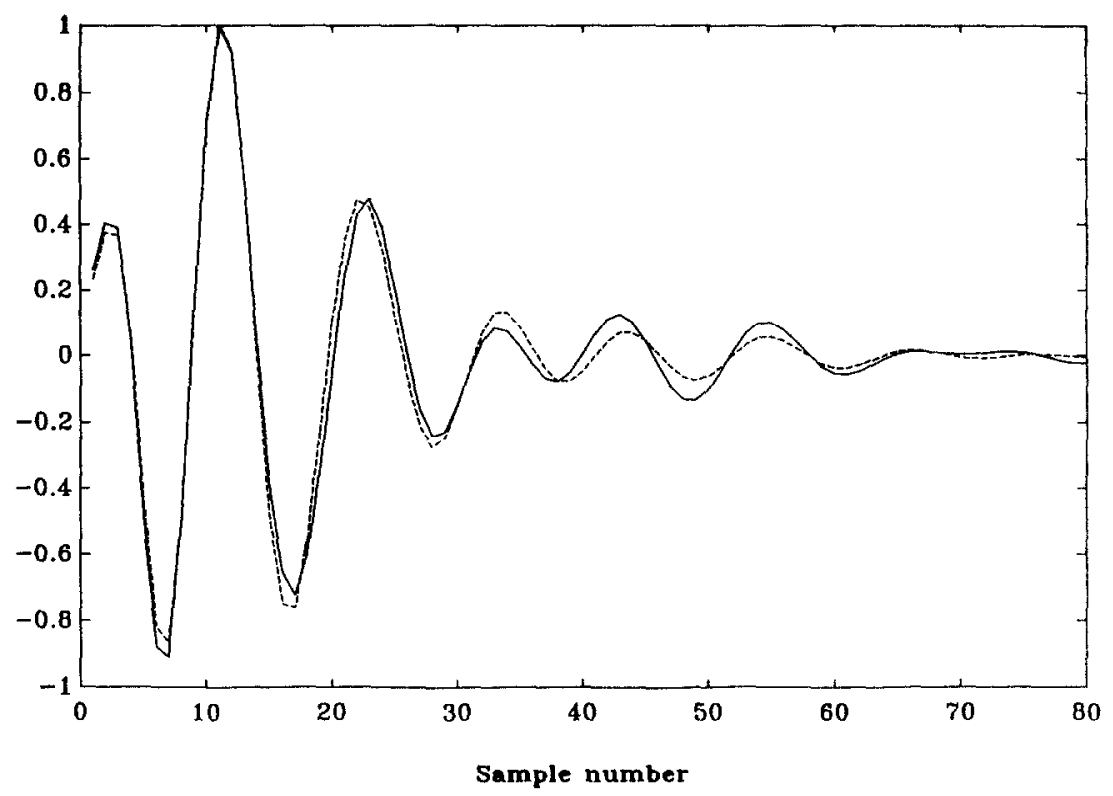

Fig. 2 Estimated wavelet when $S N R=100$.

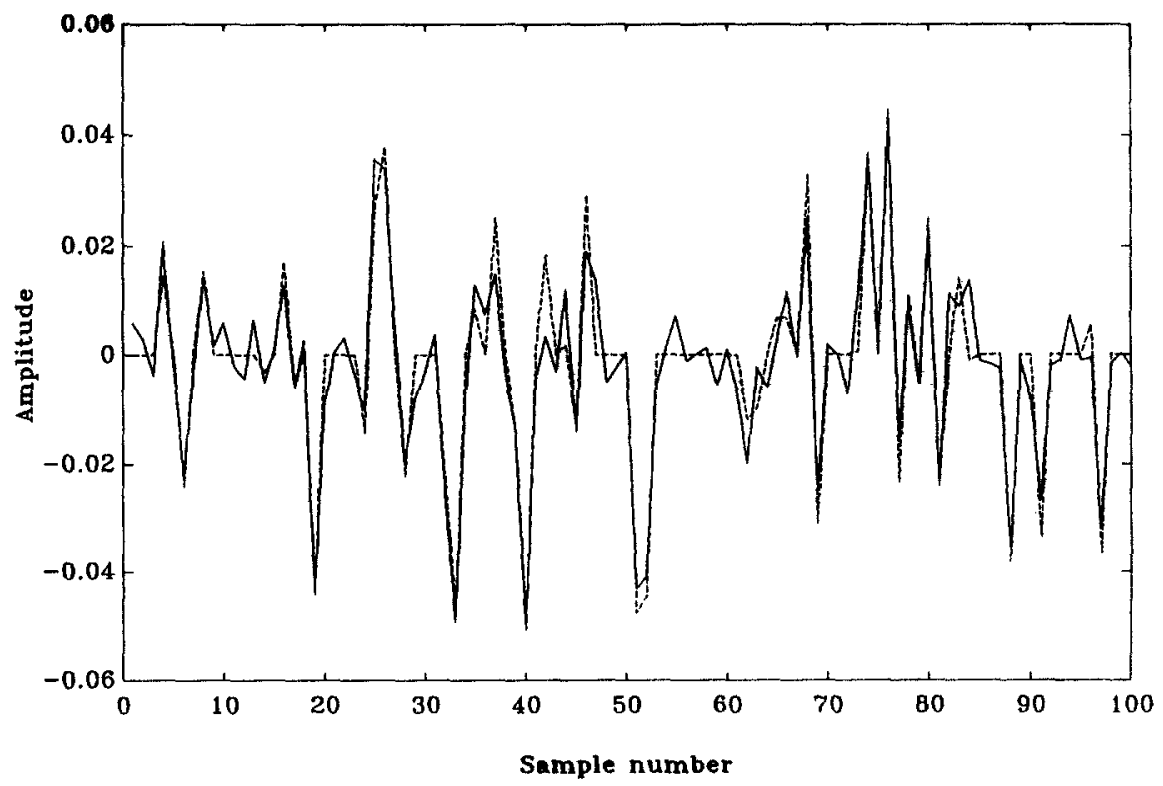

Fig. 3 Estimated and true reflection signal. $S N R=100$. 
The phantom scanned contains a substrate that generates a typical backscattered speckle signal. The data were acquired from a region of the phantom by performing a scan with a rotating transducer. Data were acquired by our special sampling system $[23,24]$. This has a resolution of 12 bits and a sampling frequency of $20 \mathrm{MHz}$. The angle between each pulse-echo line is 0.7 degrees, and 31 lines were acquired from a distance of 50 to $70 \mathrm{~mm}$ from the transducer surface.

A Brüel \& Kjær 1846 ultrasound scanner generated and received the pulses to and from the Brüel \&. Kjær type 8529 transducer. Its focal point is at $10 \mathrm{~cm}$, the nominal frequency is $3 \mathrm{MHz}$ and the diameter of the aperture is $16.2 \mathrm{~mm}$.

The covariance of the reflections was found by the pulse estimation algorithm. The covariance of the noise was found from a measurement of the noise of the total measuring system, when the transducer was submerged in a water tank. The variation in noise covariance with the time gain setting in the scanner was then tabulated and the covariance in the phantom image was calculated from this. By this method, the $Q / R_{n}$ ratio was estimated to be 4.9 , and the RMS signal-to-noise ratio was estimated to be 20.9 .

This method for determining $Q$ and $R_{n}$ is acceptable as only a small part of the image is used, where the speckle pattern is homogeneous. Further, is it has been shown that small deviations in $Q / R_{n}$ from the true ratio does not alter the deconvolved image significantly [1].

The wavelet was again estimated by using an A-line containing only a speckle signal. An $\operatorname{ARMA}(6,5)$ model was used and the resulting wavelet is shown in figure 4 .

The influence on the appearance of the image, when deconvolution has been performed, can be seen in figure 5 . The images show the logarithmic envelope of the signals. The envelope was found by Hilbert transformation. The image at the top is the normally processed image as displayed on a modern scanner.

Figure 5 shows that the average axial speckle size has decreased considerably; visually estimated to be a factor of 2 to 3 increase in resolution. This more fine grained speckle pattern

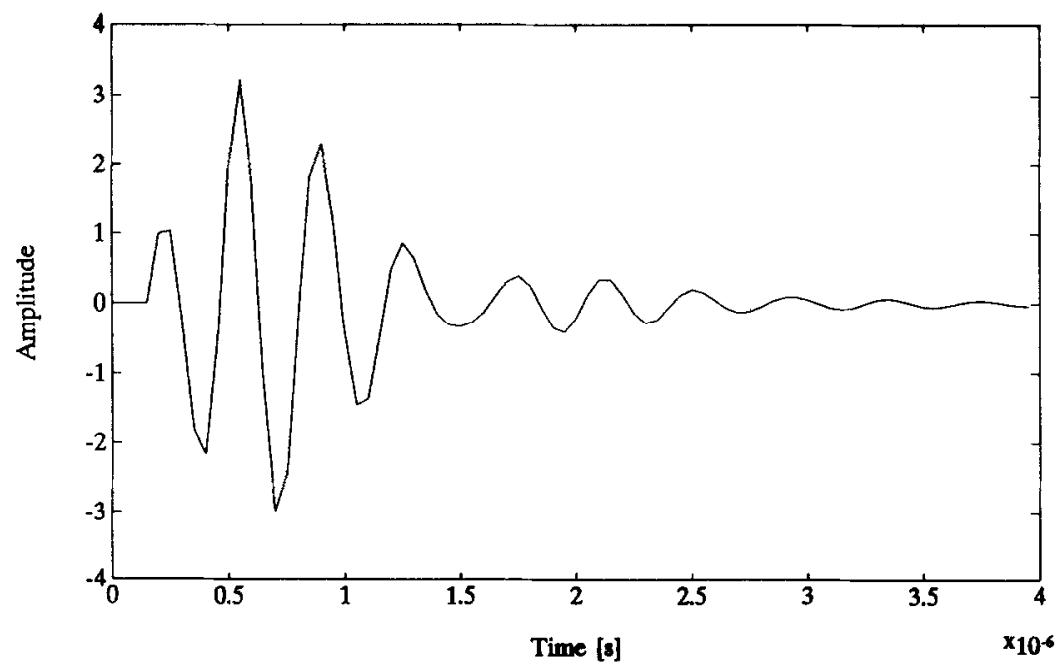

Fig. 4 Estimated wavelet for the 8529 Brüel \& Kjær transducer. 


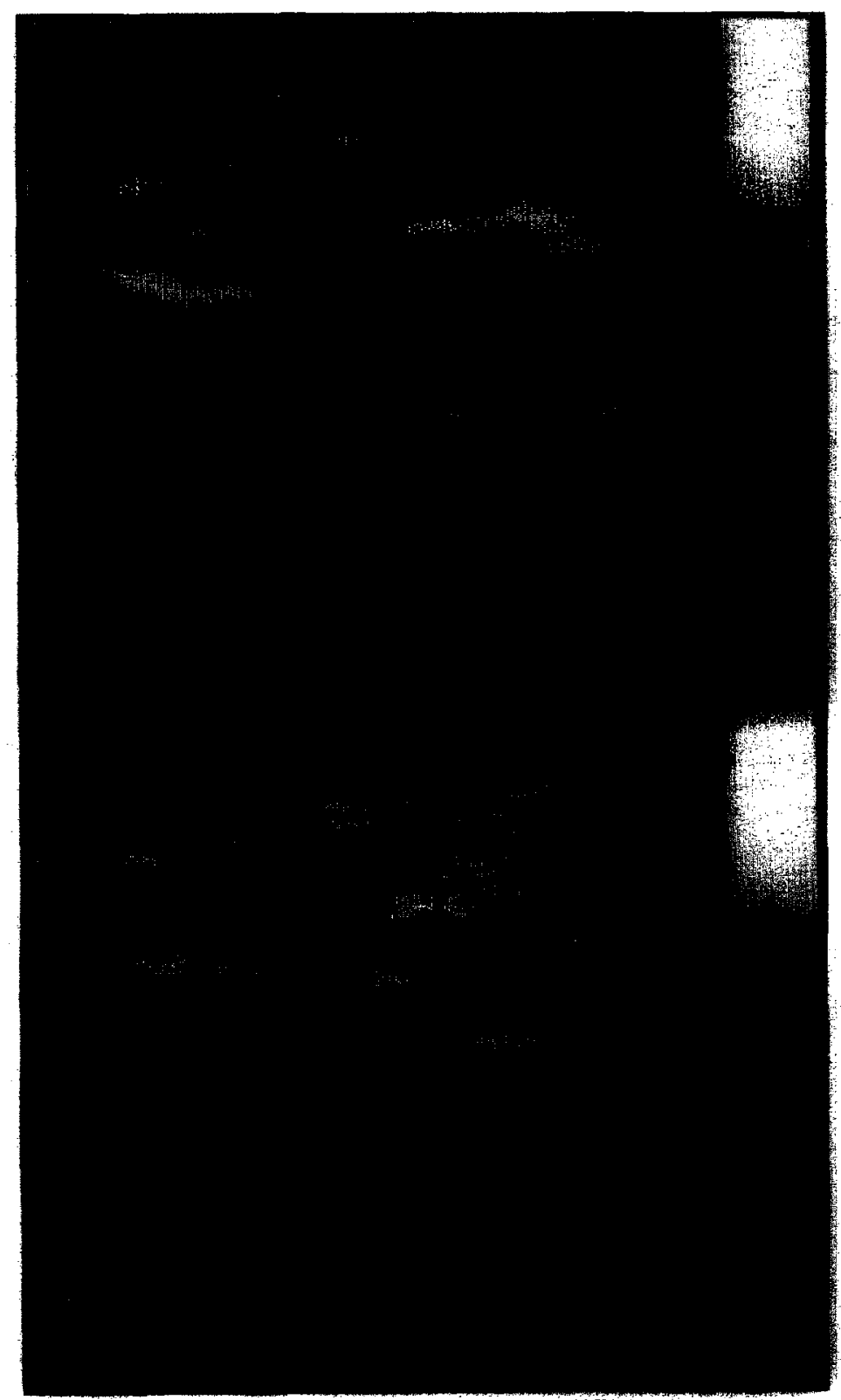

Fig. 5 Normal (top) and deconvolved (bottom) response for image measured by the Brüel \& Kjær 8529 transducer. The images cover $2 \times 2 \mathrm{~cm}$.

makes it easier to differentiate small veins and structures in the body from the random interference pattern of speckle. Also, boundaries and organ demarcations are more accurately positioned as the speckle invasion of darker areas in the images are reduced. 
One point worth noting is the slight change in resolution throughout the image. Careful examination reveals a slightly larger axial speckle size at the top and bottom of the image. This is due to the use of one set of pulse parameters for the whole image, where the pulse is essentially the mean pulse over the whole A-line. As $v_{p e a}$ is slowly changing, a better result could be obtained by segmenting the A-line and estimating parameters for each segment. Such spatially changing parameters can be handled optimally by the suggested deconvolution algorithm.

The changes in resolution also points to the importance of the pulse estimation, indicating that the pulse must and can be estimated from the actual data.

\section{SUMMARY}

Ultrasound emitted into the body is scattered by small density and propagation velocity perturbations in the tissue. Assuming linear propagation, weak reflection and scattering, and further neglecting absorption and multiple scattering, resulted in a convolution model. The received signal can be described a temporal convolution between the amplitude reflection signal originating from impedance perturbations and a spatial convolution with the pulse echo spatial impulse response of the transducer. So, the solution of the wave equation explicitly shows the influence of the transducer geometry, the pulse-echo wavelet and the tissue.

An optimal algorithm, in the least-squares sense, based on Mendel's deconvolution algorithm to estimate the amplitude reflection signal, was given. This can cope with both time-varying and two-dimensional pulses and can take into account the dispersive attenuation and noise. Further, at least in theory, it can be used for real-time image processing as it uses a fixed, predeterminable number of calculations.

Simulations involving synthetic data and an estimated wavelet revealed that the amplitude reflection signal is estimated with good precision, when the signal-to-noise ratio is sufficiently high. At a signal-to-noise ratio above 100 , both the wavelet and the reflections and their sign could be estimated well from synthetic data.

The deconvolution algorithn with wavelet estimation was used on data from a tissuemimicking phantom that generates a typical speckle pattern. A considerable increase in resolution was obtained in the axial direction, making the speckle pattern more fine grained. This makes it easier to distinguish small anatomical structures from the random speckle pattern and makes boundaries sharper.

Further research is needed to reveal the clinical usefulness of the concept. We are currently in the process of applying the algorithm to clinical, in vivo data and modifying it for the purpose. The results will be published in the future.

Still lacking is the development of a parameterization of the pulse-echo spatial impulse response. The accuracy of the physical model reported in [3] indicates that the model can be based on the analytic expression. Then no new estimation procedures need be introduced. Work is in progress along these lines to make a full two-dimensional deconvolution.

\section{ACKNOWLEDGMENT}

The research was partly funded by the Danish Technical Research Council, grant 16-4218.E, Brüel \& Kjær A/S, Novo's Foundation, H.C. Orsteds Foundation and Trane's Foundation. Dr. Sidney Leeman, King's College, London UK is thanked for proofreading the manuscript. Danish Phantom Service produced the tissue mimicking phantom used. 


\section{REFERENCES}

[1] Jensen, J.A., Medical Ultrasound Imaging, An Estimation Based Approach, Ph.D. dissertation, (Electronic Institute, Technical University of Denmark, September, 1988).

[2] Chernov, L.A., Wave Propagation in a Random Medium, (McGraw-Hill, 1960).

[3] Jensen, J.A., A model for the propagation and scattering of ultrasound in tissue, J. Acoust. Soc. Amer. 89, 188-191 (1991).

[4] Tupholme, G.E., Generation of acoustic pulses by baffled plane pistons, Mathematika 16, 209-224 (1969).

[5] Stepanishen, P.R., The time-dependent force and radiation impedance on a piston in a rigid infinite planar baffle, J. Acoust. Soc. Am. $\{9,841-849$ (1971).

[6] Stepanishen, P.R., Transient radiation from pistons in a infinite planar baffle, J. Acoust. Soc. Am. $49,1627-1638$ (1971).

[7] Ljung, L., System Identification. Theory for the User, (Prentice-Hall Inc., 1987).

[8] Norton, S.J. and Linzer, M., Ultrasonic reflectivity imaging in three dimensions; exact inverse scattering solutions for plane, cylindrical, and spherical apertures, IEEE Trans. Biomed. Eng. BME-28, 202-220 (1981).

[9] Vezzetti, D.J. and Aks A., Reconstructions from scattering data: analysis and improvements of the inverse born approximation, Ultrasonic Imaging 1, 333-345 (1979).

[10] Mendel, J.M. and Kormylo, J., New fast optimal white-noise estimators for deconvolution, IEEE Trans. Geo. Elec. GE-15, 32-41 (1977).

[11] Mendel, J. M., Optimal Seismic Deconvolution. An Estimation Based Approach (Academic Press, New York, 1983).

[12] Wiener, N., Extrapolation, Interpolation and Smoothing of Stationary Time Series, with Engineering Applications (Wiley \& Sons, Inc., New York, 1949).

[13] Fatemi, M. and Kak, A.C., Ultrasonic B-scan imaging: theory of image formation and a technique for restoration, Ultrasonic Imaging 2, 1-47 (1980).

[14] Liu, C.N., Fatemi, M. and Waag, R.C., Digital processing for improvement of ultrasonic abdominal images, IEEE Trans. Med. Imag. MI-2, 66-75 (1983).

[15] Robinson, D.E. and Wing, M., Lateral deconvolution of ultrasonic beams, Ultrasonic Imaging 6, 1-12 1984 .

[16] Hundt, E.E. and Trautenberg, E.A., Digital processing of ultrasonic data by deconvolution, IEEE Trans. Sonics Ultrasonics SU-27, 249-252 (1980).

[17] Herment, A., Demoment, G. and Vaysse, M., Algorithm for On Line Deconvolution of Echographic Signals, in Acoustical Imaging, vol. 10, P. Alais and A.F. Metherell, eds., 325-345 (Plenum Press, New York, 1980).

[18] Vollmann, W., Resolution enhancement of ultrasonic B-scan images by deconvolution, IEEE Trans. Sonics Ultrasonics SU-29, 78-83 (1982).

[19] Demoment, G., Reynaud, R. and Herment, A., Range resolution improvement by a fast deconvolution method, Ultrasonic Imaging 6, 435-451 (1984). 
[20] Kuc, R.B., Application of Kalman filtering techniques to diagnostic ultrasound, Ultrasonic Imaging 1, 105-120 (1979).

[21] Jensen, J.A., Estimation of pulses in ultrasound B-scan images, IEEE Trans. Med. Imag. MI-10, 164-172 (1991).

[22] Anderson, B.D.O. and Moore, J.B., Optimal Filtering (Prentice-Hall, Inc., New York, 1979),

[23] Jensen, J.A. and Mathorne, J., A sampling system for clinical ultrasound images, Proc. Med. Imag. V Symposium, (International Society for Optical Engineering, San Jose, California, February-March 1991).

[24] Mathorne, J. and Jensen, J.A., A sampling system for clinical ultrasound pictures, (Report C.1, Electronics Laboratory, Technical University of Denmark, 1989). 